\title{
Masked Clozapine-induced Cardiomyopathy
}

\author{
Charles A. Pastor, MD, and Monica Mehta, PharmD
}

A 33-year-old Latin-American woman with a history of psychosis and mood disorder, but no cardiac history or risk factors, presented with heart failure after $\mathbf{6}$ weeks of clozapine and olanzapine therapy. Her presentation was ambiguous and further complicated by a highly suggestible nature, which delayed the proper diagnosis and treatment. After discontinuing the antipsychotic agents and completing an otherwise negative comprehensive work-up, her heart function significantly improved (left ventricular ejection fraction increased from $38 \%$ to $53 \%$ ). A literature search showed that cardiomyopathy secondary to antipsychotics has been reported but remains poorly understood. This is the second documented case report of clozapine-induced cardiomyopathy in a Latin-American woman. (J Am Board Fam Med 2008; 21:70-74.)

On 25 April 2006, a 33-year-old Latin-American woman with a medical history of schizophrenia and bipolar disorder was transferred from an inpatient psychiatry floor to the hospital's medicine unit because she was experiencing tremors and fever. On the inpatient psychiatry floor, she was being treated for active psychosis. The patient had been taking clozapine for nearly 5 weeks and olanzapine for 6 weeks at titrating doses. Before the transfer she was receiving clozapine $100 \mathrm{mg}$ by mouth (PO) every morning, clozapine $150 \mathrm{mg}$ PO every evening, and olanzapine $20 \mathrm{mg}$ PO twice daily (BID). No other medications were being administered. The patient denied any toxic habits. There was an extensive history of psychosis in the family but no premature coronary heart disease. She is a recent immigrant from Puerto Rico, where she was also treated for her psychosis. The patient claimed to have stopped all her home medications of divalproex sodium, lithium, lorazepam, and clonazepam weeks before admission, but this was unverifiable.

This article was externally peer reviewed.

Submitted 3 April 2007; revised 30 July 2007; accepted 7 August 2007.

From the Family Medicine Department, Bronx-Lebanon Hospital Center (CAP) and the Department of Pharmacy Practice, Arnold \& Marie Schwartz College of Pharmacy and Health Sciences, Long Island University (MM), Bronx, New York.

Funding: none.

Conflict of interest: none declared.

Corresponding author: Charles A. Pastor, MD, Department of Family Medicine, Bronx-Lebanon Hospital Center, 1276 Fulton Ave, 3rd Floor, Bronx, NY 10456 (E-mail: cpastor@bronxleb.org).
During the physical examination, fever (a onetime Tmax of $100.4^{\circ} \mathrm{F}$ ), tachycardia (heart rate, 136 beats/min), and nonspecific tremors were the only positive findings. After normalizing the elevated creatinine phosphokinase (1275 units/L) with intravenous hydration and ruling out a suspected pulmonary embolism by spiral computed tomography scan, the presenting symptoms were considered fairly benign side effects from the antipsychotics. Clozapine was continued at the same dose (100 $\mathrm{mg}$ every morning and $150 \mathrm{mg}$ every evening), but olanzapine was decreased to $20 \mathrm{mg}$ at the hour of sleep. Because the fever resolved and the tremors were considered benign, the patient was transferred back to the psychiatry floor for further monitoring.

\section{Case Report}

On 2 May 2006, the patient was transferred from the psychiatry floor back to the hospital medicine floor because of persistent tremors and worsening mental status. The review of systems was negative for fever, chills, upper respiratory symptoms, shortness of breath, chest pain, palpitations, dyspnea during exertion, paroxysmal nocturnal dyspnea, paresis, dysuria, and seizure activity. The physical examination was positive for tachycardia (heart rate, 117 beats/min), diaphoresis, nonspecific asymmetrical resting tremor, ataxia, and reported visual hallucinations. No orthostasis was noted. At admission the differential diagnosis, focusing on the decompensated mental status, included delirium, neuroleptic malignant syndrome, extrapyramidal 
syndrome, infection (meningitis, HIV-related diseases), and clozapine-induced cardiopathology.

In the laboratories, creatinine phosphokinase was elevated (394 units/L) with unremarkable erythrocyte sedimentation rate and cardiac enzymes (Troponin-T and CK-MB\%). The electrocardiogram showed sinus tachycardia. Other labs (complete blood count, chem-20, thyroid-stimulating hormone, urinalysis, and arterial blood gas) were within normal limits and the urine toxicology screen was negative. In addition, the blood cultures, chest radiograph, as well as magnetic resonance imaging and computer tomography scan of the brain were unremarkable. The patient refused an HIV screening test.

The consulting psychiatrist concluded that the patient's apparent decompensating mental status, tremors, and incontinence were probably manifestations of a "highly suggestible and easily influenced nature." The patient's status vacillated from being fully functional to paralyzed and actively hallucinating without any clearly definitive neurological examination or reasonable correlation to the management. Observation and cognitive-behavioral therapy assisted in the rapid resolution of the tremors, incontinence, abnormal gait, and reported hallucinations. This behavioral issue provided an additional challenge to the medical team in properly diagnosing the patient.

Clozapine and olanzapine were discontinued 2 May 2006. An echocardiogram done 2 days after clozapine was stopped revealed a left ventricular ejection fraction of $38 \%$ with global hypokinesis, diastolic dysfunction, and normal heart geometry. The subsequent 6-day echo showed no significant change. Ten days after the antipsychotics were stopped and replaced with clonazepam $0.5 \mathrm{mg}$ PO $\mathrm{BID}$, the patient clinically improved. She was subsequently transferred to the psychiatry unit on conservative heart failure management, which included follow-up with cardiology and enalapril $2.5 \mathrm{mg}$ PO daily. Her tachycardia was controlled on metoprolol tartrate $50 \mathrm{mg}$ PO BID. The subsequent 1-month follow up echocardiogram showed an improved left ventricular ejection fraction of $53 \%$ and no other significant abnormalities.

\section{Epidemiology}

Based on a literature review in 2005, Merril et al concluded that the risk of potentially fatal cardio- myopathy or myocarditis is low and that the greatest risk is in the first month of therapy. ${ }^{1}$ The Physician's Desk Reference reports the rate of clozapine-induced cardiomyopathy in the United States as 8.9 per 100,000 person-years. $^{2}$ The Committee on Safety of Medicines in the United Kingdom reported one case in a 5000 patient sample $(0.02 \%){ }^{3}$ A review by Kilian et al reported 8 cases of clozapine-associated cardiomyopathy out of 8000 patients in Australia (0.1\%). ${ }^{4}$ Large differences in prevalence may be because of differences in reporting rates as well as characteristics of the sample size among other factors. Novartis reported $18 \%$ mortality in the 178 reported cases of cardiomyopathy. ${ }^{5}$ Myocarditis, however, has a higher incidence and worse prognosis, with a $38 \%$ fatality rate. $^{5}$ A PubMed literature search using search terms as "cardiomyopathy," "clozapine," and "case report" resulted in 23 articles. Of these 23 articles, 10 were reports of myocarditis, 6 of cardiomyopathy, 2 of pericarditis, 1 of myopericarditis, and 1 of myocardial disease. One case from 1996 described symptomatic cardiomyopathy after clozapine use in a patient with no previous cardiac history. ${ }^{6}$ Another similar report describes a 31-year-old white man who developed sinus tachycardia, but no other cardiac symptoms, after taking clozapine for 6 years. ${ }^{7}$ The aforementioned case is remarkable because of the extensive duration of clozapine use before the development of cardiomyopathy. A case from Korea described significant physical improvement after removal of clozapine and, on rechallenging the patient, subsequent recurrence of dilated cardiomyopathy. ${ }^{8}$ Furthermore, a case report of myocardial disease in a Brazilian patient taking clozapine was published in $2005,{ }^{9}$ thus making our case the second published report about a woman from Latin America. Although we understand very little about how race may have played a role in the disease occurrence or progression, it is worth noting because of the possibility of pharmacogenomic influences.

\section{Discussion}

Clozapine, a tricyclic dibenzodiazepine-derived atypical antipsychotic, is notorious for adverse effects, including, most notably, agranulocytosis. It was this life-threatening effect that delayed Food and Drug Administration approval for decades, until 1990. Nonetheless, it became the first of the 
atypical antipsychotics. These newer agents are characterized by a high ratio of serotonin to dopamine activity and mesolimbic pathway-centered dopamine antagonism. ${ }^{10}$ Consequently, atypical antipsychotics are less likely to cause extrapyramidal syndrome, tardive dyskinesia, and hyperprolactinemia. These benefits, although noteworthy, are coupled with serious limitations for clozapine. In addition to agranulocytosis, other manufacturer black-box warnings include seizures, myocarditis, and cardiomyopathy. Recently, the Food and Drug Administration mandated all antipsychotics to include a warning of "increased mortality in elderly patients with dementia-related psychosis" based on a 1.5 -fold increase in risk observed in various studies. ${ }^{5}$ Moreover, adverse effects are numerous and include autonomic dysfunction (tachycardia, hyperthermia) and neuroleptic malignant syndrome.

Despite warnings, clozapine has gained popularity because of its less-frequent extrapyramidal side effects, efficacy in schizophrenic patients who do not respond or are intolerant of other antipsychotics, and demonstrated reduction in the rate of suicides in schizophrenic patients. ${ }^{11}$ In up to one third of schizophrenic patients resistant to other neuroleptics, clozapine has proven to be effective. ${ }^{12} \mathrm{Clo-}$ zapine has also reduced the number of suicides in schizophrenics 6-fold. ${ }^{13}$ Additional studies have suggested that clozapine reduces cigarette smoking, alcohol abuse, and illicit drugs use, thereby reducing the risk for cardiovascular disease. ${ }^{14}$

Our case of cardiomyopathy is most likely iatrogenic, which is supported by 3 observations. First, the patient lacked cardiac risk factors. Secondly, signs and symptoms resolved after discontinuation of the offending agent, clozapine. The patient received both clozapine and olanzapine before developing cardiomyopathy, but implicating olanzapine is less convincing. A literature search for antipsychotics and cardiomyopathy yields almost exclusively cases of clozapine-induced disease. In addition, a 2001 data-mining study revealed a strong association between clozapine and cardiomyopathy as well as myocarditis. Some cases implicated chlorpromazine, fluphenazine, haloperidol, and risperidone, but a true association warrants further investigation for these agents. ${ }^{15}$ Lastly, cardiopathology secondary to clozapine was the most likely remaining answer after other possible etiologies were ruled out. The wide differential based on extraneous symptoms (eg tremors), which were later identified as psychiatric in nature, delayed the team from discovering the cardiomyopathy. The criteria for neuroleptic malignant syndrome was not met because the patient's only recorded fever could be explained as a benign side effect of clozapine. Her sporadic change in mental status, incontinence, and nonspecific tremor resolved with verbal therapy. Her elevated creatinine phosphokinase could also be explained by her persistent self-induced tremors. Neuroleptic malignant syndrome is a diagnosis of exclusion and low on our differential list in light of the other viable explanations for her signs and symptoms. Myocarditis was not suspected as the underlying pathology because inflammatory markers, such as erythrocyte sedimentation rate, were negative and leukocytosis was absent.

Cardiomyopathy is a weakening of the heart muscle or a change in heart muscle structure. It is often associated with inadequate heart pumping or other heart function abnormalities. ${ }^{16}$ Causes of cardiomyopathy range from idiopathic, genetic, immune, and infection to alcohol, toxins, and medications. ${ }^{17}$ Medications that are linked to cardiomyopathy include various chemotherapeutic agents (particularly anthracyclines), antiretrovirals, phenothiazines, chloroquine, and clozapine. The sequelae of clozapine-induced cardiomyopathy has remained a mystery since the first reported case in $1986 .{ }^{6}$ One theory links cardiomyopathy to accumulated effects of antipsychotic agents, some of which have alphaadrenergic blocking properties. However, this is an unlikely mechanism because cardiomyopathy is not as strongly linked to other antipsychotic agents. ${ }^{4}$ Merrill and associates hypothesize a mechanism similar to anthracycline-induced cardiomyopathy with direct cardiotoxic effects. ${ }^{1}$ Devarajan et al propose various other mechanisms to explain clozapine-induced cardiomyopathy and myocarditis. These mechanisms include: (1) an underlying deficiency of hepatic enzymes (CYP 450 to $1 \mathrm{~A} 2$ and CYP 450 to $1 \mathrm{~A} 3$ ), thereby increasing clozapine concentrations; (2) cardiotoxic effects of eosinophilic-mediated blockage of cholinergic M2 receptors; and/or (3) atmospheric ozone levels incurring dysfunctional cholinergic receptors. ${ }^{17,18}$ Promising early studies suggest the lack of antioxidative protection secondary to a low level of selenium in schizophrenics as an explanation for clozapine associated cardiomyopathy. ${ }^{19}$ The first documented 
case of cardiomyopathy associated with the use of clozapine in East Asia was in 2006 despite long use of the antipsychotic, thereby possibly implicating race and genetics. However, explanations such as different mechanisms of metabolism and possible under-reporting in East Asia have been offered. ${ }^{8}$

\section{Conclusions and Recommendations}

The patient's convoluted presentation required the team to peel away the layers of distractions. Could the diagnosis of cardiomyopathy have been made earlier? Based on the clues, should the assessment and plan have been different? Antipsychotic-induced cardiomyopathy, although well documented, can be ambiguous in presentation, as it was in our case. The patient's initial presentation of tremors and tachycardia suggested a simple case of fairly benign antipsychotic side effects. Furthermore, does a suspicion of cardiomyopathy warrant discontinuation of antipsychotics in an actively psychotic patient?

The patient's initial presentation did not suggest underlying heart failure. The only presenting symptom indicating possible cardiomyopathy was tachycardia, a very common and generally benign side effect of clozapine. Because the patient was young and healthy, thereby most likely having a high cardiac reserve, she was able to tolerate such a high degree of left ventricular dysfunction without deteriorating and manifesting signs and symptoms of overt cardiac failure. This could also explain the unremarkable chest radiograph (absence of plural effusion or abnormal cardiac silhouette). Had these signs been present, the diagnosis of cardiomyopathy could have been made earlier. The presence of tachycardia in a patient receiving clozapine should result in a workup, including an electrocardiogram and possibly an echocardiogram, based on the suspicion of this insidious pathology. The clinician should include iatrogenic cardiomyopathy or myocarditis in the differential diagnosis and evaluate the risk-to-benefit ratio of continuation of the medication, even in young and otherwise healthy patients.

Clozapine-induced cardiomyopathy is a diagnosis of exclusion that calls for practioners to be diligent in their patients' care. Not enough data has been collected to devise a viable standardized screen for clozapine-induced cardiomyopathy. Al- though the prevalence stated above is relatively low, the impacts of the disease are severe; therefore, vigilance surrounding clozapine-induced cardiomyopathy is warranted. Before initiating clozapine or any other antipsychotic, an appropriate history and physical (including a screening for possible existing underlying cardiac problems) is advisable. A family history including questions pertaining to premature coronary heart disease and schizophrenia with an associated cardiac problem should be assessed. Furthermore, obtaining a complete list of medications is also encouraged to avoid drug interactions that may cause more severe adverse reactions of antipsychotic agents. Although intuitively reasonable, no studies have supported baseline labs or cardiac studies to be done before initiating an antipsychotic. Presently, the benefits outweigh any present need to stop the use of clozapine altogether. However, if cardiopathology is suspected, stopping the antispychotic should be considered. As stated before, a differential including clozapineinduced cardiomyopathy should be a guide to initiate the workup that reasonably includes an electrocardiogram and possible echocardiogram based on the level of suspicion.

We'd like to acknowledge Remigius Okea, MD, and Jose A. Lopez, MD, MPH.

\section{References}

1. Merrill DB, Dec W, Goff D. Adverse cardiac effects associated with clozapine. J Clin Psychopharmacol 2005;25:32-41.

2. Physicians' desk reference, 59th ed. Stamford (CT): Thompson PDR; 2005.

3. Committee on Safety of Medicines. Myocarditis with antipsychotics: recent cases with clozapine (Clozaril). Current Problems in Pharmacovigilance 1993;19:910.

4. Kilian J, Kerr K, Lawrence C, Celermajer D. Myocarditis and cardiomyopathy associated with clozapine. Lancet 1999;354:1841-5.

5. Novartis. Clozaril (clozapine tablets). Prescribing information. Available at http://www.novartis.ca/ downloads/en/products/clozaril scrip e.pdf. Accessed 20 February 2007.

6. Leo R, Kreeger J, Kim K. Cardiomyopathy associated with clozapine. Ann Pharmacother 1996;30:603-5.

7. Tanner M, Culling W. Clozapine-associated dilated cardiomyopathy. Postgrad Med J 2003;79:412-3.

8. Roh S, Ahn D, Nam J, Yang B, Lee B, Kim Y. Cardiomyopathy associated with clozapine. Exp Clin Psychopharmacol 2006;14:94-8. 
9. De Boni R, Gama CS, Lobato MI. [Mocardial disease in a patient treated with clozapine.] Rev Bras Psiquiatr 2005;27:164.

10. Worrel J, Marken P, Beckman S, et al. Atypical antipsychotic agents. Am J Health Syst Pharm 2000; 57:238-58.

11. Sungwon R, Dong H, Jung H, Byung H, Bang H, Yang S. Cardiomyopathy associated with clozapine. Exp Clin Psychopharmacol 2006;14:94-8.

12. Conley RR. Optimizing treatment with clozapine. J Clin Psychiatry 1998;59:44-8.

13. Walker AM, Lanza LL, Arellano F, et al. Mortality in current and former users of clozapine. Epidemiology 1997;8:671-7.

14. Green A, Burgess E. Use in schizophrenia: effects of clozapine vs. risperidone. Schizophr Res 2003;60: $81-5$.
15. Coulter D, Bate A, Meyboom R, et al. Antipsychotic drugs and heart muscle disorder in international pharmacovigilance: data mining study. BMJ 2001; 322:1207-9.

16. Berger A, Medline Plus. (2006) Cardiomyopathy. Available at http://www.nlm.nih.gov/medlineplus/ ency/article/001105.htm. Accessed 20 February 2007.

17. Wehmeier P, Heiser P, Remschimidt H. Myocarditis, pericarditis and cardiomyopathy in patients treated with clozapine. J Clin Pharm Ther 2005;30: 91-6.

18. Devarajan S, Kutcher SP, Dursun SM. Clozapine and sudden death [Letter]. Lancet 2000;355:841.

19. Vaddadi K, Soosai E, Vaddadi G. Low blood selenium concentrations in schizophrenic patients on clozapine. Br J Clin Pharmacol 2003;55:307-9. 